\title{
Topic and audience effects on distinctively Scottish vocabulary usage in Twitter data
}

\author{
Philippa Shoemark* \\ p.j.shoemarkeed.ac.uk \\ *School of Informatics \\ University of Edinburgh
}

\author{
James Kirby $^{\dagger}$ \\ j.kirby@ed.ac.uk
}

\author{
Sharon Goldwater* \\ sgwater@inf.ed.ac.uk
}

\author{
$\dagger$ Dept. of Linguistics and English Language \\ University of Edinburgh
}

\begin{abstract}
Sociolinguistic research suggests that speakers modulate their language style in response to their audience. Similar effects have recently been claimed to occur in the informal written context of Twitter, with users choosing less region-specific and non-standard vocabulary when addressing larger audiences. However, these studies have not carefully controlled for the possible confound of topic: that is, tweets addressed to a broad audience might also tend towards topics that engender a more formal style. In addition, it is not clear to what extent previous results generalize to different samples of users. Using mixedeffects models, we show that audience and topic have independent effects on the rate of distinctively Scottish usage in two demographically distinct Twitter user samples. However, not all effects are consistent between the two groups, underscoring the importance of replicating studies on distinct user samples before drawing strong conclusions from social media data.
\end{abstract}

\section{Introduction}

Linguistic variation in social media is a growing research area, with interest stemming both from the engineering goal of developing tools that work well across different styles and dialects (Hovy, 2015; Stoop and van den Bosch, 2014; Vyas et al., 2014; Huang and Yates, 2014), and from the social science goal of studying user behaviour (Bamman et al., 2014; Eisenstein, 2015; Huang et al., 2016; Nguyen et al., 2015). However, this type of research is often complicated by the messy nature of social media data, which can make it hard to control for different explanatory factors and to know whether results obtained on a particular user sample generalize to another sample.

For example, previous studies have suggested that Twitter users modulate their use of regional and non-standard language depending on the expected size of the audience (operationalized as whether a Tweet contains hashtags, @-mentions, or neither) (Pavalanathan and Eisenstein, 2015a; Shoemark et al., 2017). However, these studies did not sufficiently control for possible effects of topic, which may be confounded with audience size: e.g., users may use more hashtags when discussing political events than when discussing daily routines. These studies also did not look at the degree to which their results generalize across different populations of users.

In this work we study two largely disjoint groups of (mainly) Scottish Twitter users: one group sent tweets geotagged within Scotland, while the other used hashtags related to the 2014 Scottish independence referendum. We use mixed-effects models to tease apart the effects of audience and topic on their choice of Scottish-specific terms. We find that in both user groups, topic and audience have independent effects on the rate of Scottish usage, providing stronger evidence than in previous work that users are indeed sensitive to their audience.

Nevertheless, our study does not confirm all aspects of previous work. When comparing our two user groups, the effect of topic is qualitatively similar: tweets about lifestyle or politics have lower rates of Scottish usage than "chitchat" tweets. However, the effects of audience differ between the two groups. For the geotagged users, rates of Scottish usage follow the pattern predicted by previous research: lowest among tweets with the largest expected audience, and rising as the expected audience size shrinks. In contrast, the independence referendum group showed a less consistent and less pronounced pattern which does not align cleanly 
with audience size. We were unable to find a clear explanation of this difference. Nevertheless, it highlights the difficulty of sampling representative groups from social media data and the need to interpret results with caution until they are shown to generalize across several different populations.

\section{Background}

Bell's (1984) Audience Design theory posits that intra-speaker stylistic variation is primarily conditioned by the audience of the interaction. Bell argues that stylistic variation across topics derives from so-called 'reference groups' whom the speaker associates with the topics in question, and predicts that effects of topic on style variation will be weaker than direct effects of audience. However, later studies of spoken conversation (e.g. Rickford and McNair-Knox, 1994) have suggested that both topic and audience affect a speaker's style, and that topic may even have a greater effect. Topic also appears to influence stylistic variation in computermediated communication - for example, statistical associations between lexical features and author attributes such as gender are often mediated by the topic of discourse (Herring and Paolillo, 2006; Bamman et al., 2014).

Our work is primarily inspired by two previous studies of Twitter users and how their use of regional lexical variants is influenced by either audience (Pavalanathan and Eisenstein, 2015a) or topic (Shoemark et al., 2017). In the first of these, Pavalanathan and Eisenstein (2015a) studied lexical items that were strongly associated with tweets from specific regions of the US, as determined by a data-driven approach (Eisenstein et al., 2011). They found that users were less likely to use these regional terms, as well as other nonstandard terms, in tweets containing hashtags, and more likely to do so in tweets containing @-mentions (i.e., other users' IDs). They attributed these findings to styleshifting in relation to audience size, since tweets with hashtags are more likely to be viewed by users outside of the author's follower group, while by default tweets which begin with a mention are shown only to the author, the mentioned user, and their mutual followers.

While suggestive, there are alternative explanations for this finding. For example, in their study of Scottish tweets, Shoemark et al. (2017) pointed out that if users use the word 'masel' (a Scottish variant of standard English 'myself') less frequently in tweets with hashtags, it could be simply because people talk about themselves less in tweets with hashtags, not because they are modulating the use of a regionally specific variant.

Shoemark et al. (2017) focused mainly on effects of topic rather than audience, but to avoid similar confounds, they measured the frequencies of regional variants of lexical variables ${ }^{1}$ relative to their standard variants. They found that, amongst users who tweeted about the Scottish independence referendum, both pro- and anti-independence users decreased their use of Scottish-specific terms in tweets containing referendum-related hashtags, compared to other tweets. A follow-up analysis suggested that this effect might be due to the larger audience obtained by using referendum-related hashtags, but the evidence was indirect as the original study was not designed to test that hypothesis.

Our work extends these two previous studies by building models that include factors for both topic and audience. We follow Shoemark et al. (2017) in focusing on variables that alternate between Scottish English and Standard English variants, but use a wider range of topics identified with a topic model rather than just hashtags. We use mixed-effects logistic regression in order to establish whether there are independent effects of audience and topic, whilst controlling for variation in the base rate of Scottish-variant usage across different users and variables. In addition, we explicitly examine how different methods of sampling users might affect results, by performing the same study on two user groups gathered in different ways.

\section{Data}

\subsection{Lexical variables}

We use 50 of the 51 lexical variables identified by Shoemark et al. (2017). Each variable consists of one or more distinctively Scottish variants and one or more Standard English variants, all of which are referentially and syntactically equivalent; examples are shown in Table 1. From the original 51 variables, we discard SHIT, since the variant identified as Scottish-specifc, SHITE, is used at a higher rate than the Scottish-specific forms of the other variables (e.g. 27\% of SHIT occurences in Shoemark et al.'s Indyref-Tweets dataset are realized as SHITE; more than twice the rate of Scottish variant use for any other variable), and for many users SHIT is the

\footnotetext{
${ }^{1} \mathrm{~A}$ variable is any linguistic item than can be produced in different ways; the variants are the different realizations.
} 


\begin{tabular}{lll}
\hline Variable & Scottish variants & Std variants \\
\hline DONT & DEH, DINI, DINNY & DONT, DO NOT \\
FOOTBALL & FITBA & FOOTBALL \\
MYSELF & MASEL, MASELF & MYSELF \\
SOMETHING & SUHIN & SOMETHING \\
TO & TAE & TO, TOO \\
\hline
\end{tabular}

Table 1: Examples of lexical variables.

only variable for which any Scottish variant use is observed. This suggests that SHITE is less marked as 'distinctively Scottish' than the Scottish-specific variants of the other 50 variables.

\subsection{Dataset construction}

We aim to study Scottish language use, but only a small proportion of Twitter users disclose their location, either by including it in their user profile or by opting to automatically tag their tweets with geographic coordinates when using a GPS-enabled device. Moreover, studies have indicated that those who do share their location are not representative of the wider Twitter user base (Pavalanathan and Eisenstein, 2015b; Sloan and Morgan, 2015).

To help assess the generalizability of our findings, we therefore consider two datasets, both covering the same time period but sampled from distinct (though slightly overlapping) populations: 'Scottish Geotag Users', who have tagged their tweets with locations in Scotland; and 'Indyref Hashtag Users', who have used hashtags relating to the 2014 Scottish Independence Referendum. As we will demonstrate, users in the two samples do differ in some aspects of their behaviour, emphasizing how biases implicit in the construction of datasets can affect results.

Our two groups of users are taken from the Geotagged-Scotland (GS) and Indyref-Tweets (IT) datasets collected by Shoemark et al. (2017). Both of these datasets were drawn from an archive of Twitter's 'Spritzer' stream, which provides a 1\% sample of the public data flowing through Twitter, covering the period from September 2013 to September 2014. The GS dataset consists of tweets by users for whom the archive contained at least one tweet which was geotagged with a location in Scotland, while the IT dataset consists of users for whom it contained at least one tweet with a hashtag relating to the 2014 Scottish Independence referendum (see Table 3 in Shoemark et al. (2017) for a list of hashtags).

As a heuristic to filter out bots and spammers,

\begin{tabular}{ll|ll} 
& & IH Users & SG Users \\
\hline (a) & N Users & 14,572 & 17,942 \\
& N Tweets & $4,703,040$ & $1,750,343$ \\
& N Variables & $10,482,683$ & $3,733,133$ \\
& \% Scottish & 0.5 & 1.8 \\
\hline (b) & N Users & 12,101 & 11,307 \\
& N Tweets & $4,674,251$ & $1,678,498$ \\
& N Variables & $10,424,067$ & $3,594,659$ \\
& \% Scottish & 0.5 & 1.8 \\
\hline (c) & N Users & 10,786 & 10,103 \\
& N Tweets & $3,456,277$ & $1,371,694$ \\
& N Variables & $7,689,621$ & $2,878,352$ \\
& \% Scottish & 0.7 & 2.3 \\
\hline (d) & N Users & 10,784 & 10,103 \\
& N Tweets & $2,165,320$ & $1,112,931$ \\
& N Variables & $4,934,186$ & $2,365,496$ \\
& \% Scottish & 0.8 & 2.3
\end{tabular}

Table 2: Dataset statistics for Indyref Hashtag Users and Scottish Geotag Users (a) after basic preprocessing, (b) after discarding users with $<50$ variable instances, (c) after discarding users for which there is strong evidence of non-use of Scottish variants and (d) after labelling audience \& topic. '\% Scottish' is the percentage of variables realized as the Scottish variant.

we computed the proportion of tweets for each user in the GS and IT datasets which contained URLs, and discarded users for whom this proportion was in the 90th percentile. For the remaining users, we then retrieved a more complete set of their tweets: for each user we attempted to retrieve all the tweets they posted in August, September, or October 2014 (excluding retweets), using Twitter's REST API. The API allows us to retrieve up to 3200 of a user's most recent tweets, so if a user had posted more than 3200 tweets since autumn 2014, we were unable to retrieve their tweet histories for this period. We obtained complete histories for at least one of the three months for a total of 18,370 Scottish Geotag (SG) Users, and 14,832 Indyref Hashtag (IH) Users. We then applied some simple ad-hoc text filters to remove tweets produced by apps which automatically share user's horoscopes or track users' follower counts, as well as some particularly prevalent types of marketing tweets. See Table 2a for summary statistics after this filtering step. Note that there are 363 users who are in both datasets.

Next, we removed all users for whom the total number of observed variable instances was less than 50 (see Table 2b), as with so few observations it would be difficult to make reliable inferences about these users' usage rates of distinctively Scot- 
tish variants.

Finally, since our population of interest is those who vary between Scottish and standard variants, we discard individuals for whom we had enough observed variable instances to conclude that they probably never used distinctively Scottish variants of any of our variables. For SG Users, we chose the threshold of 'enough observed variable instances' to be 298 , since this is the smallest value $n$ such that the cumulative binomial probability of seeing at least one Scottish variant in $n$ variable instances is $\geq 0.99$ (assuming a constant usage rate of Scottish variants of 0.0184 , as listed in Table $2 b$ ). That is, if we assume that any user who does use Scottish variants will do so $1.84 \%$ of the time, then in $99 \%$ of cases where we have observed at least 298 variable instances from such a user, we would expect a Scottish variant to have been used in at least one of those instances. For IH Users, we assumed a constant usage rate of distinctively-Scottish variants of 0.05 , and discarded all those for whom we had observed at least 870 variable instances and no Scottish variants. Table 2c provides summary statistics for the two resulting datasets.

When considering the differences in average rates of Scottish variant usage across the two groups, it is important to note that Shoemark et al. (2017) identified these Scottish variants using the GS dataset, i.e. the same dataset from which we drew our Scottish Geotag Users. It is therefore to be expected that that the Scottish Geotag Users would use these variants at a higher rate, and it is important to bear in mind that the Indyref Hashtag Users may be more frequent users of other distinctively Scottish variants.

\section{Topic \& Audience}

\subsection{Audience labelling}

We follow Pavalanathan and Eisenstein (2015a) in assuming that tweets containing hashtags (any token prepended with the '\#' character) typically have a wider audience than other tweets, since anyone interested in a particular topic or event can browse the stream of Tweets which contain associated hashtags. Conversely, tweets beginning with @-mentions typically have a narrow audience since by default they only appear in the feeds of the author, the mentionee, and users who follow both the author and the mentionee. Any user @-mentioned in a tweet (whether at the beginning, or elsewhere within the tweet) will by default receive a special

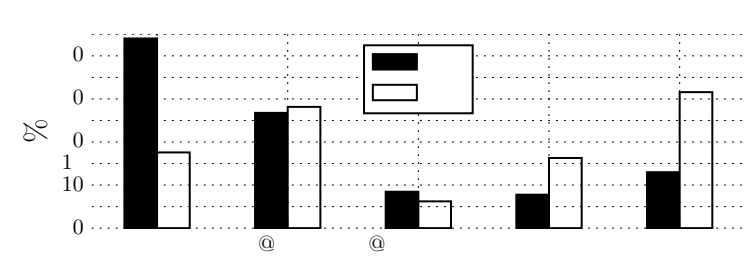

Figure 1: Distribution of tweets with each audience label in the two datasets.

notification drawing their attention to it.

Pavalanathan and Eisenstein hypothesise that both kinds of mention serve to narrow the intended audience, whilst hashtags serve to widen it, relative to broadcast tweets (i.e., those without hashtags or mentions, which appear on the feeds of all the author's followers). The grounds for hypothesising a narrowing function for tweet-internal mentions are less evident than those for tweet-initial mentions, since tweets which do not begin with a mention are not limited by default to the feeds of the author and mentionee's mutual followers.

We label each variable instance in our two datasets with three binary variables indicating whether or not they contain hashtags, initial mentions, and/or internal mentions. We then discard any tweets for which two or more of these indicators are activated, since we do not have intuitive a priori hypotheses about how combining more than one of these variables within a single tweet would affect its intended audience.

Figure 1 shows the proportion of tweets in each dataset which have each audience label (or which had multiple labels and were subsequently discarded), and reveals qualitative differences in the two groups' behaviour: SG Users post relatively more 'broadcast' tweets, whilst IH Users use relatively more hashtags (which is unsurprising given that they were selected on the basis of their hashtag use).

\subsection{Topic labelling}

We assign topics to tweets using a Latent Dirichlet Allocation (LDA) topic model (Blei et al., 2003) estimated with collapsed Gibbs sampling (Griffiths and Steyvers, 2004) from both datasets combined. Following Hong and Davison (2010) and others, we create 'documents' by concatenating together tweets by the same author. To account for possible topic drift within individuals over time, we group each user's tweets by month and model each peruser-per-month document as a distinct mixture of 
topics. We use the inferred topic model parameters to label each tweet with a topic, as described below.

The corpus was preprocessed as follows: tweets were tokenised using the Twokenize program ${ }^{2}$, a tokeniser designed specifically for Twitter text, and all non-alphabetic tokens, except for those which begin with hashtags, were discarded. The vocabulary was then pruned to the 100,000 most frequent terms across the two datasets. We set the number of topics, $T$, to 30 , and used symmetric Dirichlet priors of $\alpha=\frac{50}{T}$ and $\beta=0.01$ on the multinomial distributions over topics and terms, respectively ${ }^{3}$. The Gibbs sampler was run for 750 iterations.

Upon inspection of the most probable words and documents for each topic, we deemed that twenty of the topics could be grouped into three broader themes, which we describe as 'chatter' , 'lifestyle', and 'politics' . Later, we consider a different grouping, where we split off a 'sports' theme from the 'lifestyle' theme, and an 'indyref' theme from the 'politics' theme. Table 3 shows the most probable words (excluding stopwords) for each topic within these three/five themes. Of the ten topics that we did not assign to these themes, four could be described as spam topics, four as foreign language, and two as relating to purely stylistic dimensions as opposed to any particular topic of discussion: one for distinctively Scottish terms, and the other for 'netspeak'-style spellings and abbreviations.

To assign topic labels to individual tweets, we take a Gibbs sample and then for a given tweet, each topic $t$ is assigned a weight, defined as

$$
\text { weight }_{t}=\sum_{w \in \boldsymbol{w}} \hat{p}(t \mid w)
$$

where $\boldsymbol{w}$ is the bag of words which occur in the tweet (excluding stopwords and any variant of any of our variables of interest), and $\hat{p}(t \mid w)$ is obtained by maximum likelihood estimation from the Gibbssampled topic-token assignments. Finally, we take the topic with the highest weight, and label the tweet with its broader theme. If the topic with the highest weight is one of the two 'stylistic' topics, we defer to the topic with the next highest weight. We discard tweets labelled as 'spam' or 'foreign language', as well as those for which the highest weight is not unique, if the topics which share this weight belong to different themes.

\footnotetext{
${ }^{2}$ https://github.com/myleott/ark-twokenize-py

${ }^{3}$ During development we experimented with values for $\mathrm{T}$ between 10 and 100 , and $\alpha$ between 0.015 and 1.5 , and saw little qualitative difference in the themes that emerged, based on manual inspection of topic keywords.
}

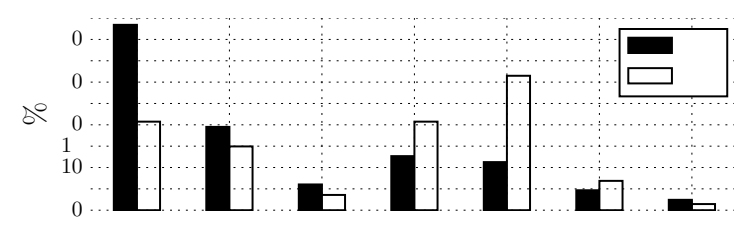

Figure 2: Distribution of tweets with each topic label in the two datasets.

Using this method, we obtain $2.3 \mathrm{~m}$ broad-topiclabeled variable instances from SG Users, and $4.9 \mathrm{~m}$ from IH Users. Figure 2 shows the distribution of topics in each data set, and Table 4 gives a breakdown of variable instances by audience-type and broad-topic-label. IH Users have a much larger proportion of tweets with 'indyref' or 'politics' labels than SG Users, which once again is unsurprising, given how these users were sampled.

\section{Method}

We use the glmer() function from the lme4 package (Bates et al., 2015) for R (R Core Team, 2013) to estimate mixed effects logistic regression models, predicting Scottish variant usage (yes $=1$, no $=0$ ) from the intended audience size and topic of the tweet in which a lexical variable occurs. Our fourlevel categorical audience factor (initial mention, internal mention, broadcast, hashtag) is dummy coded into three binary variables, with broadcasts as the reference level. Our tweet topic labels are also dummy coded, taking the 'chatter' topic as the reference level. By specifying random effects for users and variables, we control for the influence of different baseline rates of Scottish variant usage across different users and variables. Hence our models are of the form

$$
\operatorname{logit}\{E(\mathbf{y})\}=\mathbf{X} \boldsymbol{\beta}+\mathbf{Z u}, \mathbf{y} \sim \text { Bernoulli }
$$

where $\mathbf{y}$ is the $n \times 1$ vector of responses from a Bernoulli distribution, $\mathbf{X}$ is an $n \times p$ design matrix for the fixed effects $\boldsymbol{\beta}$, and $\mathbf{Z}$ is an $n \times q$ design matrix for the random effects $\mathbf{u}$. We do not include random slopes in our models, since we do not have enough observations per group to provide stable estimates of the variances. Our models are fit by Laplacian approximation to Maximum Likelihood estimation. 


\begin{tabular}{|c|c|}
\hline Topic theme & Keywords \\
\hline \multirow{4}{*}{ Chatter } & love feel life fucking fuck people shit actually hate omg school gonna time excited oh \\
\hline & time yeah bit oh probably actually maybe seen lot pretty hope haha bad getting stuff \\
\hline & lol love thank xx thanks hope day oh happy lovely xxx ha haha morning beautiful \\
\hline & night happy birthday haha day wait tonight tomorrow hahaha bed getting wee weekend days week \\
\hline \multirow{6}{*}{ Lifestyle } & $\begin{array}{l}\text { love song music album world amazing god top white black girl watch band ice looks baby life listen } \\
\text { guy boys }\end{array}$ \\
\hline & $\begin{array}{l}\text { photo watching \#xfactor \#cbb day \#scotland loving posted \#gbbo life \#glasgow \#bbuk \#love \#edinburgh } \\
\text { love }\end{array}$ \\
\hline & video \#auspol liked game awesome watch time apple iphone play app games phone buy facebook \\
\hline & oh bit news ha twitter story brilliant bbc read book called tv look dear wonder \\
\hline & day time morning night car run food bit nice week train getting tea eat days \\
\hline & $\begin{array}{l}\text { tonight day week time tomorrow night glasgow morning looking edinburgh forward coming weeks } \\
\text { hear live }\end{array}$ \\
\hline \multirow{2}{*}{ Sports } & cup win ireland \#glasgow2014 irish time team final match scottish round top games race live \\
\hline & game celtic team football season league fans mate goal win play players club player haha \\
\hline \multirow{6}{*}{ Politics } & people read agree question thanks issue debate political article course mean change indeed etc politics \\
\hline & news police pm russia minister russian via eu report ukraine president ebola court uk \#ukraine \#russia \\
\hline & \#ferguson rt obama \#ukraine police \#cdnpoli ukraine video via mt people news american time america \\
\hline & labour uk ukip cameron party tory ed tax vote tories english mps miliband boris david \\
\hline & people lol look tell money time stop wrong please believe mean job care saying talking \\
\hline & israel \#gaza war via isis gaza \#isis world people children israeli \#israel police hamas support \\
\hline \multirow[t]{2}{*}{ Indyref } & $\begin{array}{l}\text { \#indyref scotland \#voteyes \#yes vote scottish independence \#scotdecides \#indyrefpic \#bettertogether } \\
\text { salmond \#bbcindyref \#the45 campaign debate }\end{array}$ \\
\hline & scotland vote uk labour scottish snp scots union oil party wm country westminster voters voting \\
\hline
\end{tabular}

Table 3: Topic themes and the top 15 keywords for each topic within each theme

\begin{tabular}{ll|r|r|r|r} 
& Topic & Chatter & Lifestyle & Politics & All \\
\hline (a) & Broadcast & $598,673(2.7)$ & $334,143(2.3)$ & $295,981(1.8)$ & $1,228,797(2.4)$ \\
& Initial Mention & $352,981(3.0)$ & $164,909(2.9)$ & $188,191(1.9)$ & $706,081(2.7)$ \\
& Internal Mention & $92,682(1.8)$ & $63,242(1.5)$ & $56,727(1.2)$ & $212,651(1.6)$ \\
& Hashtag & $67,630(1.8)$ & $69,833(1.4)$ & $80,504(1.2)$ & $217,967(1.4)$ \\
All & $1,11,966(2.7)$ & $632,127(2.3)$ & $621,403(1.7)$ & $2,365,496(2.3)$ \\
\hline \hline (b) & Broadcast & $308,797(1.3)$ & $341,592(0.9)$ & $658,520(0.8)$ & $1,308,909(1.0)$ \\
& Initial Mention & $644,459(1.1)$ & $394,036(1.0)$ & $1,026,634(0.6)$ & $2,065,129(0.8)$ \\
& Internal Mention & $76,403(0.6)$ & $96,123(0.5)$ & $203,275(0.4)$ & $375,801(0.5)$ \\
& Hashtag & $124,333(0.7)$ & $197,925(0.5)$ & $862,089(0.5)$ & $1,184,347(0.5)$ \\
All & $1,153,992(1.1)$ & $1,029,676(0.8)$ & $2,750,518(0.6)$ & $4,934,186(0.8)$
\end{tabular}

Table 4: Counts of variable instances in the (a) SG Users and (b) IH Users datasets, broken down by Topic and Audience. In each cell, the percentage of variable instances that are Scottish variants is given in parentheses. 


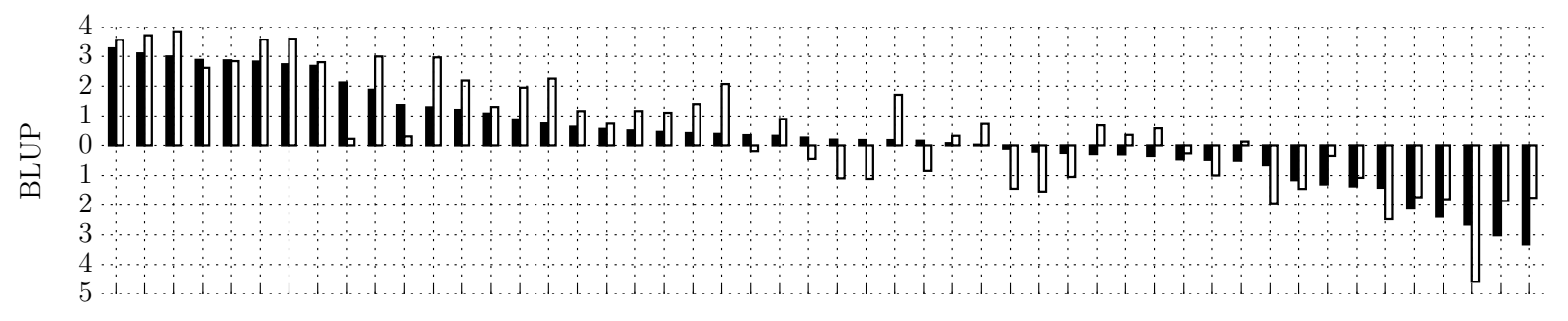

Figure 3: Barplots of by-variable BLUPs for SG Users (black bars) and for IH Users (white bars).
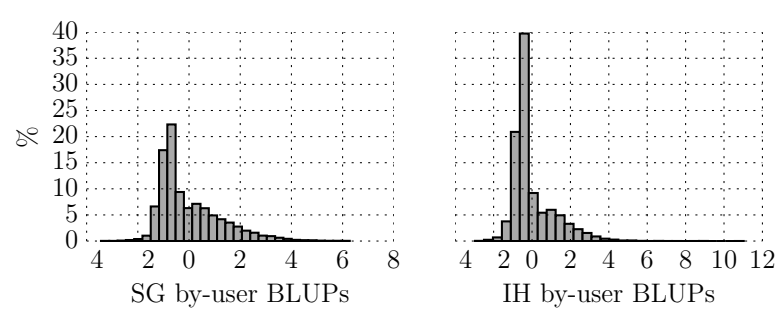

Figure 4: Histograms of by-user BLUPs.

\section{Results and Discussion}

\subsection{Random Intercepts}

We begin by constructing null models that predict the log odds of choosing a Scottish variant using only intercepts, which we allow to vary randomly by each user and by each lexical variable. The estimated variances of the by-user and by-variable adjustments to the intercept are given in Table $5 \mathrm{a}$, for SG and IH Users, respectively.

The Best Linear Unbiased Predictors (BLUPs) of the by-variable random intercerpts (i.e. the posterior estimates, given the data and model parameters, of the adjustment to the intercept for each variable) are shown in Figure 3. In both datasets, open class variables (e.g. GRANDAD, BALLS, DOGS) tend to have higher rates of Scottish variant usage than closed class variables (e.g. WAS, OF, YOU).

Figure 4 shows the distributions of by-user BLUPs. Although the models assume a normal distribution over the by-user intercepts, the BLUPs are positively skewed. We suspect the BLUPs reflect the fact that our datasets contain a mixture of two populations: Scottish speakers, who use Scottish variants at a range of rates, and non-Scottish speakers, who rarely if ever use Scottish variants. The non-Scottish speakers are responsible for the large number of users with slightly negative intercepts. Unfortunately there is no straightforward way to separate these groups (especially for users with a relatively small number of observations). However, users with a constant near-zero rate of Scottish variant usage should, at worst, dilute any true effects of topic or audience on rates of usage, but should not change the direction of those effects.

\subsection{Random Intercepts + Audience Effects}

We now check whether Pavalanathan and Eisenstein's (2015a) reported effects of hashtags and mentions on the odds of using regional variants in US tweets, are replicated for distinctively Scottish variants in our two datasets.

We augment our null models with our dummycoded audience factors as fixed effects. For each dataset, we assess the goodness-of-fit using chisquare tests on the log-likelihoods. Compared to the null models with only random effects, including fixed effects for audience significantly improves the fit on both datasets (SG: $\chi 2(3)=643.05, \mathrm{p}=$ $<2.2 \mathrm{e}-16$; IH: $\chi 2(3)=232.69, \mathrm{p}=<2.2 \mathrm{e}-16)$.

Parameters of the models with Audience effects are in Table 5b. Our results for SG Users largely accord with those of Pavalanathan and Eisenstein (2015a): Scottish variants are positively associated with tweet-initial mentions, and negatively associated with hashtags. Relative to broadcast tweets, the odds of seeing Scottish variants are about $28 \%$ higher in tweets with initial mentions, and about $17 \%$ lower in tweets with hashtags. However, while Pavalanathan and Eisenstein also found an association between the use of tweet-internal mentions and local/non-standard words in their data, our model does not show such a relationship in the SG dataset.

In the IH dataset, the audience effects in our model do not follow the pattern that Pavalanathan and Eisenstein observed in US tweets. Unlike for SG Users, there is no association between hashtags and Scottish variants, and the effects of mentions are in the opposite direction to those found by Pavalanathan and Eisenstein (2015a). Amongst 
IH Users, initial mentions are negatively associated with Scottish variants, though the effect size is very small. Internal mentions are also negatively associated with Scottish variants, and in this case the effect is relatively large (in contrast with SG Users, for whom we found no effect). We discuss possible reasons for this result in Section 6.4.

\subsection{Random Intercepts + Topic Effects}

Next, we test for a relationship between the topic of a tweet and the odds of Scottish variant usage. For both datasets, models with fixed effects for topic significantly improve the fit over random-effectsonly models (SG: $\chi 2(2)=570.48, \mathrm{p}=<2.2 \mathrm{e}-16$; IH: $\chi 2(2)=1241, \mathrm{p}=<2.2 \mathrm{e}-16)$.

Parameters of the models are in Table $5 \mathrm{c}$. The effects of tweet topic are qualitatively similar in each dataset: relative to 'chatter' tweets, tweeting about the 'lifestyle' topic reduces the odds of choosing Scottish variants by $11 \%$ for SG Users and 5\% for IH Users, while tweeting about politics reduces them by $27 \%$ for SG Users, and $39 \%$ for IH Users.

\subsection{Full Models}

For each dataset, including fixed effects for audience and topic together significantly improves the model fit, both over the models with fixed effects for audience only (SG Users: $\chi 2(2)=508.67, \mathrm{p}=$ $<2.2 \mathrm{e}-16$; IH Users: $\chi 2(2)=1298.9, \mathrm{p}=<2.2 \mathrm{e}-$ 16), and over the models with fixed effects for topic only (SG: $\chi 2(3)=581.25, \mathrm{p}=<2.2 \mathrm{e}-16$; IH: $\chi 2(3)$ $=290.6, \mathrm{p}=<2.2 \mathrm{e}-16)$.

Parameters of the full models are in Table $5 \mathrm{~d}$. When fixed effects for audience and topic are included together in the SG model, their effect sizes barely change. These results suggest that for SG Users, audience and topic have independent effects on Scottish usage, and that even after accounting for topic, the effects of audience size are as predicted by Pavalanathan and Eisenstein (2015a).

In the full IH model, while most of the fixed effect sizes are relatively unchanged, a positive association between the use of hashtags and Scottish variants emerges. Thus, the model reveals that the qualitative behavior of these users is very different from that of the SG Users. Although topic and audience are both significant factors in the models for each group, initial mentions and hashtags have the opposite effects for IH Users as for SG Users (and for Pavalanathan and Eisenstein's user sample).

Although they primarily interpret their findings in terms of audience size, Pavalanathan and Eisen- stein acknowledge that mentions and hashtags can affect the composition of the audience in more nuanced ways than just its size. As an alternative explanation for the positive associations they found between mentions and local/non-standard words, they suggest that authors may use such words to express particular identities or stake claims to local authenticity, specifically when addressing users for whom such claims are meaningful.

In theory, this account could also apply to the positive association we find in the IH dataset between hashtags and local variants: while on the one hand, the indexing function of hashtags can be conceived of as broadening the audience of a tweet, on the other hand it could serve to narrow the tweet's intended audience, by explicitly targeting it at a circumscribed community. Hence, when using hashtags associated with communities for whom the notion of Scottish identity has strong currency (e.g. people with strong views on indyref, or supporters of a particular sports team), IH Users may use Scottish variants initiatively, in order to emphasise that part of their identity.

Suppose that authors tended to decrease their use of Scottish variants when discussing most political issues, but increase it when discussing Scottish independence - either to emphasise their own Scottish identity, or to accommodate towards an audience which is likely to contain many Scottish people. If this were the case, our models would be unable to account for this variation directly, since we have grouped indyref and other political issues together. However, since a large proportion (55\%) of IH Users hashtag tweets are actually about indyref, one way the IH model could account for a difference between indyref and general politics is to increase the weight for hashtags. If this were the case, then including 'indyref' as a distinct topic should improve the model fit and alleviate the impact on the audience weights. To test this hypothesis, we performed a follow-up study where we split the topics into finer-grained categories.

\subsection{Finer-grained topics}

We added two topic categories, 'sport' and 'indyref', which we split off from the 'lifestyle' and 'politics' categories, respectively (see Table 3). Contrary to our hypothesis, re-defining the topic categories in this way made little difference to the model fit: the log-likelihoods for the new full model are -174169.4 for SG Users, and -121447.8 


\begin{tabular}{|c|c|c|c|c|c|c|c|}
\hline & \multicolumn{3}{|c|}{ Scottish Geotag Users } & \multicolumn{4}{|c|}{ Indyref Hashtag Users } \\
\hline (a) & \multicolumn{3}{|c|}{$\begin{array}{l}\text { Log-likelihood: }-174758.0 \\
\sigma^{2} \text { users: } 2.769 \quad \sigma^{2} \text { variables: } 2.477\end{array}$} & \multicolumn{4}{|c|}{$\begin{array}{l}\text { Log-likelihood: }-122240.2 \\
\sigma^{2} \text { users: } 3.058 \quad \sigma^{2} \text { variables: } 3.444\end{array}$} \\
\hline (b) & \multicolumn{3}{|c|}{$\begin{array}{l}\text { Log-likelihood: }-174436.4 \\
\sigma^{2} \text { users: } 2.750 \quad \sigma^{2} \text { variables: } 2.503\end{array}$} & \multicolumn{4}{|c|}{$\begin{array}{l}\text { Log-likelihood: }-122123.9 \\
\sigma^{2} \text { users: } 3.039 \quad \sigma^{2} \text { variables: } 3.443\end{array}$} \\
\hline $\begin{array}{l}\text { Fixed Ef. } \\
\text { @init } \\
\text { @intrnl } \\
\text { hashtag }\end{array}$ & $\begin{array}{ll}\text { OR } & 95 \% C I \\
1.28 & (1.25,1.31) \\
0.96 & (0.92,1.00) \\
0.83 & (0.80,0.86)\end{array}$ & $\begin{array}{l}z \\
21.2 \\
-1.9 \\
-8.9\end{array}$ & $\begin{array}{l}|z|) \\
16 \\
2 \\
16\end{array}$ & $\begin{array}{l}\text { OR } \\
0.96 \\
0.62 \\
0.97\end{array}$ & $\begin{array}{l}95 \% C I \\
(0.93,0.99) \\
(0.59,0.67) \\
(0.93,1.01)\end{array}$ & $\begin{array}{l}z \\
-2.8 \\
-15.4 \\
-1.6\end{array}$ & $\begin{array}{l}\operatorname{Pr}(>|z|) \\
0.005 \\
<2 \mathrm{e}-16 \\
0.111\end{array}$ \\
\hline (c) & \multicolumn{3}{|c|}{$\begin{array}{l}\text { Log-likelihood: }-174472.7 \\
\sigma^{2} \text { users: } 2.758 \quad \sigma^{2} \text { variables: } 2.472\end{array}$} & \multicolumn{4}{|c|}{$\begin{array}{l}\text { Log-likelihood: }-121619.7 \\
\sigma^{2} \text { users: } 3.069 \quad \sigma^{2} \text { variables: } 3.427\end{array}$} \\
\hline & $\begin{array}{ll}\text { OR } & 95 \% \text { CI } \\
0.89 & (0.87,0.91) \\
0.73 & (0.71,0.75)\end{array}$ & $\begin{array}{l}z \\
-9.9 \\
-24.2\end{array}$ & $\begin{array}{l}\operatorname{Pr}(>|z|) \\
<2 \mathrm{e}-16 \\
<2 \mathrm{e}-16\end{array}$ & $\begin{array}{l}\text { OR } \\
0.95 \\
0.61\end{array}$ & $\begin{array}{l}95 \% C I \\
(0.92,0.98) \\
(0.59,0.63)\end{array}$ & $\begin{array}{l}z \\
-3.2 \\
-33.6\end{array}$ & $\begin{array}{l}\operatorname{Pr}(>|z|) \\
0.001 \\
<2 \mathrm{e}-16\end{array}$ \\
\hline (d) & \multicolumn{3}{|c|}{$\begin{array}{l}\text { Log-likelihood: }-174182.1 \\
\sigma^{2} \text { users: } 2.742 \quad \sigma^{2} \text { variables: } 2.496\end{array}$} & \multicolumn{4}{|c|}{$\begin{array}{l}\text { Log-likelihood: }-121474.4 \\
\sigma^{2} \text { users: } 3.063 \quad \sigma^{2} \text { variables: } 3.416\end{array}$} \\
\hline $\begin{array}{l}\text { Fixed Ef. } \\
\text { @ init } \\
\text { @intrnl } \\
\text { hashtag } \\
\text { lifestyle } \\
\text { politics }\end{array}$ & $\begin{array}{ll}\text { OR } & 95 \% C I \\
1.27 & (1.24,1.29) \\
0.96 & (0.92,1.00) \\
0.85 & (0.82,0.89) \\
0.90 & (0.88,0.92) \\
0.74 & (0.72,0.76)\end{array}$ & $\begin{array}{l}z \\
20.6 \\
-1.9 \\
-7.6 \\
-8.7 \\
-22.9\end{array}$ & $\begin{array}{l}\operatorname{Pr}(>|z|) \\
<2 \mathrm{e}-16 \\
0.052 \\
<3 \mathrm{e}-14 \\
<2 \mathrm{e}-16 \\
<2 \mathrm{e}-16\end{array}$ & $\begin{array}{l}\text { OR } \\
0.93 \\
0.63 \\
1.08 \\
0.95 \\
0.60\end{array}$ & $\begin{array}{l}95 \% C I \\
(0.90,0.95) \\
(0.60,0.67) \\
(1.04,1.12) \\
(0.91,0.98) \\
(0.58,0.61)\end{array}$ & $\begin{array}{l}z \\
-5.04 \\
-15.3 \\
3.9 \\
-3.4 \\
-34.3\end{array}$ & $\begin{array}{l}\operatorname{Pr}(>|z|) \\
<5 \mathrm{e}-07 \\
<2 \mathrm{e}-16 \\
<1 \mathrm{e}-04 \\
<0.001 \\
<2 \mathrm{e}-16\end{array}$ \\
\hline
\end{tabular}

Table 5: Summary of model parameters for the two datasets: (a) random intercepts only, (b) random intercepts + audience effects, (c) random intercepts + topic effects, (d) full model. $\sigma^{2}$ users and $\sigma^{2}$ variables are variance estimates for the random intercepts. Fixed $E f$. tables show odds ratios $(O R)$ derived from logit coefficients, with roughly estimated confidence intervals (using approximate standard errors), and results of Wald's z-tests.

for IH Users (c.f. Table 5d).

In general, the effect sizes and directions of the newly defined subtopics are similar to those of the broad topics from which they were isolated, and more importantly, changing the topic definitions has no effect on the audience coefficients for either user group. This provides some evidence that our results are not highly sensitive to the precise choice of topics.

\section{Conclusion}

This study examined how Twitter users shift their use of Scottish variants depending on the topic and audience. We looked at two groups of users with different overall rates of Scottish usage and found that both topic and audience affect usage in both groups. The qualitative effects of topic were similar across the two groups, demonstrating a clear relationship between the topic or genre of discussion and the odds of choosing Scottish variants. However, the sizes and directions of the audience affects are inconsistent across the two groups: for Scottish Geotag Users we found (as in a previous study) that local variants are used more in tweets with initial mentions and less in tweets with hashtags, but for Indyref Hashtag Users we found the opposite. The demographics and usage patterns of these two groups are very different, and one interesting possibility is that they might be using the affordances of mentions and hashtags in different ways and focusing on different aspects of how these affect their potential audience. In any case, our results underscore the need for caution when drawing broad conclusions from studies of social media data, until the results of those studies are shown to hold across a variety of user samples. 


\section{Acknowledgments}

This work was supported in part by the EPSRC Centre for Doctoral Training in Data Science, funded by the UK Engineering and Physical Sciences Research Council (grant EP/L016427/1) and the University of Edinburgh.

\section{References}

David Bamman, Jacob Eisenstein, and Tyler Schnoebelen. 2014. Gender identity and lexical variation in social media. Journal of Sociolinguistics 18(2):135160 .

Douglas Bates, Martin Mächler, Ben Bolker, and Steve Walker. 2015. Fitting linear mixed-effects models using lme4. Journal of Statistical Software 67(1):148.

Allan Bell. 1984. Language style as audience design. Language in society 13(02):145-204.

David M Blei, Andrew Y Ng, and Michael I Jordan. 2003. Latent dirichlet allocation. Journal of $\mathrm{Ma}$ chine Learning Research 3(Jan):993-1022.

Jacob Eisenstein. 2015. Systematic patterning in phonologically-motivated orthographic variation. Journal of Sociolinguistics 19(2):161-188.

Jacob Eisenstein, Amr Ahmed, and Eric P. Xing. 2011. Sparse additive generative models of text. In Proceedings of the International Conference on $\mathrm{Ma}$ chine Learning. pages 1041-1048.

Thomas L Griffiths and Mark Steyvers. 2004. Finding scientific topics. Proceedings of the National Academy of Sciences 101(suppl 1):5228-5235.

Susan C Herring and John C Paolillo. 2006. Gender and genre variation in weblogs. Journal of Sociolinguistics 10(4):439-459.

Liangjie Hong and Brian D Davison. 2010. Empirical study of topic modeling in twitter. In Proceedings of the first workshop on social media analytics. ACM, pages 80-88.

Dirk Hovy. 2015. Demographic factors improve classification performance. In Proceedings of the 53rd Annual Meeting of the Association for Computational Linguistics. Association for Computational Linguistics, pages 752-762.

Fei Huang and Alexander Yates. 2014. Improving word alignment using linguistic code switching data. In Proceedings of the 14th Conference of the European Chapter of the Association for Computational Linguistics. Association for Computational Linguistics, Gothenburg, Sweden, pages 1-9.
Yuan Huang, Diansheng Guo, Alice Kasakoff, and Jack Grieve. 2016. Understanding U.S. regional linguistic variation with Twitter data analysis. Computers, Environment and Urban Systems 59:244 - 255.

Dong Nguyen, Dolf Trieschnigg, and Leonie Cornips. 2015. Audience and the use of minority languages on twitter. In Proceedings of the Ninth International Conference on Web and Social Media. pages 666669.

Umashanthi Pavalanathan and Jacob Eisenstein. 2015a. Audience-modulated variation in online social media. American Speech 90(2):187-213.

Umashanthi Pavalanathan and Jacob Eisenstein. 2015b. Confounds and consequences in geotagged Twitter data. In Proceedings of the 2015 Conference on Empirical Methods in Natural Language Processing. Association for Computational Linguistics, pages 2138-2148.

R Core Team. 2013. R: A Language and Environment for Statistical Computing. R Foundation for Statistical Computing, Vienna, Austria. http://www.Rproject.org/.

John R Rickford and Faye McNair-Knox. 1994. Addressee-and topic-influenced style shift: A quantitative sociolinguistic study. Sociolinguistic perspectives on register pages 235-276.

Philippa Shoemark, Debnil Sur, Luke Shrimpton, Iain Murray, and Sharon Goldwater. 2017. Aye or naw, whit dae ye hink? Scottish independence and linguistic identity on social media. In Proceedings of the 15th Conference of the European Chapter of the Association for Computational Linguistics: Volume 1, Long Papers. Association for Computational Linguistics, pages 1239-1248.

Luke Sloan and Jeffrey Morgan. 2015. Who tweets with their location? Understanding the relationship between demographic characteristics and the use of geoservices and geotagging on Twitter. PloS one 10(11):e0142209.

Wessel Stoop and Antal van den Bosch. 2014. Using idiolects and sociolects to improve word prediction. In Proceedings of the 14th Conference of the European Chapter of the Association for Computational Linguistics. Association for Computational Linguistics, Gothenburg, Sweden, pages 318-327.

Yogarshi Vyas, Spandana Gella, Jatin Sharma, Kalika Bali, and Monojit Choudhury. 2014. POS tagging of English-Hindi code-mixed social media content. In Proceedings of the 2014 Conference on Empirical Methods in Natural Language Processing. volume 14, pages 974-979. 